\title{
Silicon Oxynitride Optical Waveguide Ring Resonator Utilizing a Two-Mode Interferometer Structure
}

\author{
Kaixin Chen ${ }^{1}$ and Hau Ping Chan ${ }^{2}$ \\ ${ }^{1}$ School of Communication and Information Engineering, University of Electronics Science and Technology of China, Chengdu, \\ Sichuan 610054, China \\ ${ }^{2}$ The Department of Electronic Engineering, City University of Hong Kong, Kowloon, Hong Kong \\ Correspondence should be addressed to Kaixin Chen, chenkxcn@yahoo.com.cn
}

Received 26 May 2011; Revised 17 September 2011; Accepted 3 October 2011

Academic Editor: Leonid Khriachtchev

Copyright (๑) 2012 K. Chen and H. P. Chan. This is an open access article distributed under the Creative Commons Attribution License, which permits unrestricted use, distribution, and reproduction in any medium, provided the original work is properly cited.

Silicon oxynitride $\left(\mathrm{SiO}_{x} \mathrm{~N}_{y}, \mathrm{SiON}\right)$ optical waveguide ring resonator, in which a two-mode interferometer is used to replace the directional coupler in a conventional ring resonator, has been designed and fabricated. Preliminary results exhibit the same of free spectral range of $100 \mathrm{GHz}$ but different quality factors of 3700 and 3900 at $1550 \mathrm{~nm}$ for transverse electric (TE) and transverse magnetic (TM) mode, respectively. The extinction ratio is more than $18 \mathrm{~dB}$ over the entire C-band, and the insertion loss is lower than $9.5 \mathrm{~dB}$ for TE and TM mode.

\section{Introduction}

Optical waveguide ring resonators are attracting much attention in recent years because they are most often used as a building block in optical filters [1, 2], sensing system [3, 4], laser resonator [5], and switching [6] due to their special transmission characteristics and compact configuration. Using different fabrication technology, optical waveguide ring resonators have been demonstrated with different materials: $\mathrm{Si}_{-} \mathrm{SiO}_{2}$ [7], $\mathrm{SiON}$ [8], polymer [3, 9], GaAs [10], and glass [11]. Silicon oxynitride $\left(\mathrm{SiO}_{x} \mathrm{~N}_{y}, \mathrm{SiON}\right)$ is a promising material for optical waveguide ring resonator because its refractive index can be tunable with a large range (from 1.45 to 2.0), which means it can be used to fabricate optical waveguide with a moderate index contrast $[12,13]$. Such an optical waveguide is helpful to reduce scattering loss, fiber-to-waveguide coupling loss, and tolerance sensitivity presented in optical waveguide ring resonator. In addition, its excellent optical transparence (from $210 \mathrm{~nm}$ to beyond $2000 \mathrm{~nm}$ ) allows low-loss optical waveguide to be made. Furthermore, SiON optical waveguide can be easily fabricated using standard photolithography and reactive ion etching (RIE) technique. Some SiON optical waveguide devices, such as elliptic couplers [14], ring [8] or microring [15] resonator, adaptive gain equalizer [16], and integrated spectrometers [17] have been reported.

In this paper, we present a SiON optical waveguide ring resonator, in which a two-mode interferometer (TMI) coupler is used to replace the directional coupler (DC) in a conventional ring resonator. And this is a significant difference between our SiON ring resonator and the one reported in [8], where, using TMI coupler to replace DC introduces three advantages. Firstly, the device can be designed more compact because the coupling length of TMI coupler is shorter than that of DC. Secondly, the device is with higher fabrication tolerance because the concern to control the critical gap separation in the case of a DC structure can be circumvented. Such as, by using TMI coupler to replace DC, the fabrication tolerance sensitivity reduced by a factor of 2 was reported [18]. At last, TMI coupler is less polarization dependent due to the fact that the relative mode dispersion is less polarization dependent in strongly coupled waveguides $[18,19]$. Due to the aforementioned virtue, TMI coupler is usually used as a basic component to design and fabricate a lot of integrated optical waveguide devices, such as wavelength multiplexers [20, 21], optical switch [22], and polarization splitter [23]. Using TMI coupler, we have also demonstrated optical waveguide interleaver with 
optical polymer materials $[24,25]$. In fact, TMI is very suitable for the application to replace DC used in many optical waveguide devices with high index or moderate index contrast [26], especially for the ring resonator. The fabricated device exhibits the same free spectral range of $100 \mathrm{GHz}$ but different quality factors of 3700 and 3900 at $1550 \mathrm{~nm}$ for transverse electric (TE) and transverse magnetic (TM) mode, respectively. The extinction ratio is more than $18 \mathrm{~dB}$ over the entire C-band, and the insertion loss is lower than $9.5 \mathrm{~dB}$ for TE and TM mode.

\section{Design and Fabrication of Ring Resonator}

Figure 1(a) schematically depicts the configuration of the proposed SiON optical ring resonator. The light is coupled from an input single mode waveguide into the ring resonator with a TMI. The TMI coupler is formed by connecting two Y-junctions (Y1 and Y2) back to back with a two-mode (symmetrical and antisymmetrical) waveguide trunk with interaction length $S$. Although the geometrical structure of a TMI is very similar to a four-port MMI, the operating principle is totally different. In fact, it is more like a $3 \mathrm{~dB}$ directional coupler in aspect of operating principle. When a TMI coupler is formed using the materials with high index or moderate index contrast, the coupling effect between the two branching waveguides of the two Y-junctions in the TMI can be ignored and the two modes will be created only in the two-mode waveguide trunk; therefore the interference is only carried out in this region. For this case, TMI can be treated as a pure model, that is, a zero gap DC and the transfer matrix of the TMI can be written as [26]

$$
\begin{gathered}
\left(\begin{array}{cc}
\cos \left(\frac{\Delta \varphi}{2}\right) & i \sin \left(\frac{\Delta \varphi}{2}\right) \\
i \sin \left(\frac{\Delta \varphi}{2}\right) & \cos \left(\frac{\Delta \varphi}{2}\right)
\end{array}\right) \\
\Delta \varphi=\left(\beta_{0}-\beta_{1}\right) S
\end{gathered}
$$

where $\beta_{0}$ and $\beta_{1}$ are, respectively, the propagation constants of the symmetric mode and the antisymmetric mode of the two-mode waveguide trunk. Using the notion in Figure 1(a) and (1), the coupling between the ring and the waveguide can be described by the following matrix equation:

$$
\left(\begin{array}{l}
E_{3} \\
E_{4}
\end{array}\right)=\left(\begin{array}{cc}
\cos \left(\frac{\Delta \varphi}{2}\right) & i \sin \left(\frac{\Delta \varphi}{2}\right) \\
i \sin \left(\frac{\Delta \varphi}{2}\right) & \cos \left(\frac{\Delta \varphi}{2}\right)
\end{array}\right)\left(\begin{array}{l}
E_{1} \\
E_{2}
\end{array}\right) .
$$

In view of the attenuation and the phase shift when the field propagates in the ring, we have

$$
E_{2}=\alpha E_{4} \exp (-i \beta L),
$$

where $L$ is the circumference of the ring with radius $r, \beta$ is the propagation constant in the ring waveguide, and $\alpha$ is the field amplitude attenuation constant. From (3) and (4), the transfer function of the ring resonator can be described as

$$
\left|\frac{E_{3}}{E_{1}}\right|^{2}=\frac{\cos ^{2}(\Delta \varphi / 2)-2 \alpha \cos (\Delta \varphi / 2) \cos (\beta L)+\alpha^{2}}{1-2 \alpha \cos (\Delta \varphi / 2) \cos (2 \beta L)+\alpha^{2} \cos ^{2}(\Delta \varphi / 2)} .
$$

Equation (5) implies that the resonance occurs when the condition $\beta L=2 m \pi$ is met, so the resonance wavelength $\lambda_{c}$ is then obtained as

$$
\lambda_{c}=\frac{n_{\mathrm{eff}} L}{m}
$$

where $n_{\text {eff }}$ is the effective index of the guided mode propagating in the ring waveguide and $m$ is an integer representing the resonance order. From (6), free spectral range (FSR) of ring resonator can be written as in [1].

$$
\mathrm{FSR}=\frac{c}{n_{g} L}
$$

where $n_{g}=n_{\text {eff }}+f_{0}\left(d n_{\text {eff }} / d f\right)_{f_{0}}$ is group index of the waveguide.

Figures 1(b) and 1(c) also show the materials used in our optical ring resonator, where SiON and polymer ZP51 are used as the core and the cover cladding of the waveguide, respectively. The refractive indices of SiON and ZP51 at $1550 \mathrm{~nm}$ are 1.5684 and 1.51 for TE polarization and 1.5705 and 1.5095 for TM polarization, respectively. With them, the two different waveguide geometry and dimensions in the ring resonator, that is, two-mode waveguide used in the interaction region of the TMI and single mode waveguide used in the other parts, can be determined by utilizing effective index method, and the results are also shown in Figures 1(b) and 1(c), respectively.

The radius of the ring is crucial on the rings' design because it is the most dominating parameter to determine the bending loss and the FSR of the ring. Using the parameters presented in Figure 1(c), the bending losses of ring waveguides with different radius was calculated at wavelength of $1530 \mathrm{~nm}$ and $1570 \mathrm{~nm}$ for TE and TM mode when light travels one round, and the results are shown in Figure 2, where the dispersion of the two materials is negligible and the same index for different wavelength was used in calculation. From Figure 2, although the bending losses are different for different operating wavelength and for different mode, they are negligible when the radius is larger than $200 \mu \mathrm{m}$.

Furthermore, with the refractive indices and the waveguide dimensions shown in Figures 1(b) and 1(c), together with the (6) and (7), the proposed SiON optical waveguide ring resonator with a channel spacing of $100 \mathrm{GHz}$ $(\sim 0.8 \mathrm{~nm}$ at $1550 \mathrm{~nm})$ was designed, which is suitable for a wavelength-division multiplexed system. The radius of the ring is $300 \mu \mathrm{m}$, and the bending loss is negligible with this radius. The two-mode interaction length $S$ is a crucial parameter to realize the critical coupling condition and hence high extinction ratio. The length $S$ for achieving the extinction ration more than $50 \mathrm{~dB}$ for TE and TM mode was calculated as $24.8 \mu \mathrm{m}$ and $24.2 \mu \mathrm{m}$, respectively. Therefore, a tradeoff is necessary in determining the optimal length $S$ for both modes. In our case, the length was selected to be $24.5 \mu \mathrm{m}$, which is the average value of both lengths. Finally, with aforementioned designing parameters, the transmission spectra of the designed ring resonator can be simulated using (5). 


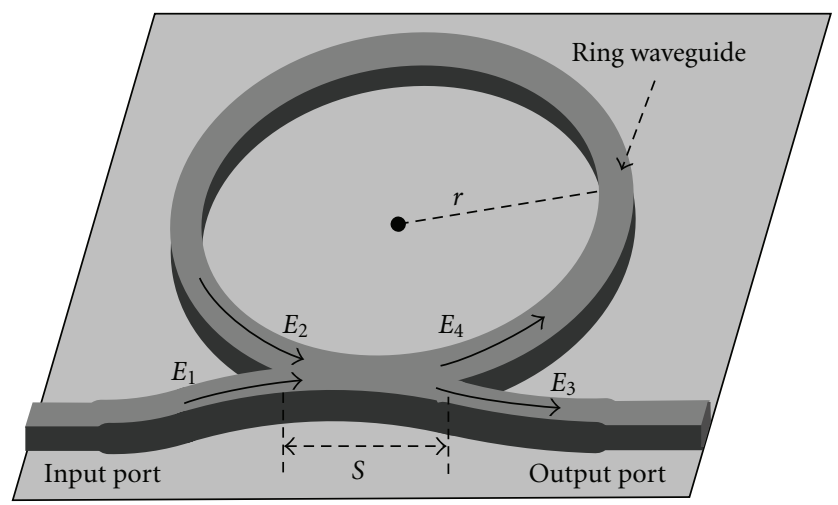

(a)

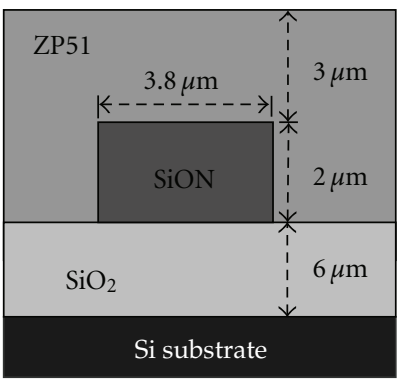

(b)

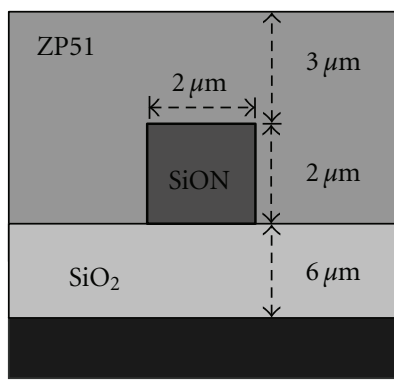

(c)

FIGURE 1: Schematic diagram of the SiON optical waveguide ring resonator (a), together with the materials and the dimensions of the two-mode waveguide (b) and the single waveguide (c) used in the fabrication work.

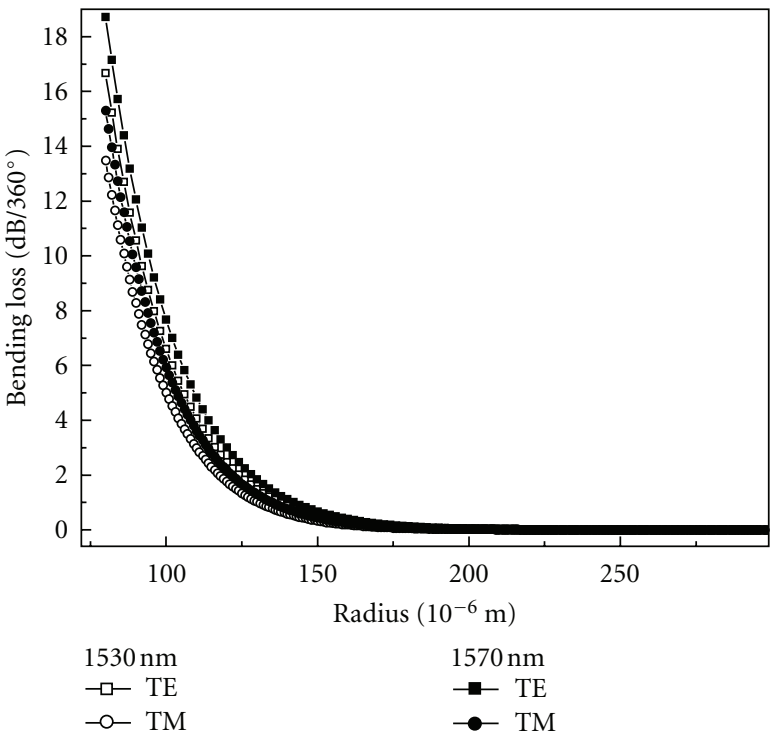

Figure 2: The bending losses per turn as a function of the ring radius at wavelength of $1530 \mathrm{~nm}$ and $1570 \mathrm{~nm}$ for TE and TM mode.

The standard photolithography and reactive ion etching (RIE) technique was used to fabricate the proposed SiON optical waveguide ring resonator. Firstly, a silicon wafer which had a layer of SiON core of $2.0 \mu \mathrm{m}$ thickness and a layer of Silicon oxide cladding of $6 \mu \mathrm{m}$ thickness was cleaned and dried in an oven. Secondly, a layer of chromium (Cr) of $50 \mathrm{~nm}$ thickness used as the mask in RIE process was deposited onto the SiON layer by radio-frequency magnetron sputtering. Thirdly, the waveguide pattern of the ring resonator was formed by the standard photolithography technique and then transferred to the Cr layer by wet etching, and it was further transferred to the SiON layer by RIE using a mixture of carbon tetrafluoride $\left(\mathrm{CF}_{4}\right)$ and oxygen $\left(\mathrm{O}_{2}\right)$. Lastly, after removing the $\mathrm{Cr}$ mask layer, a film of polymer ZP49 of $3.0 \mu \mathrm{m}$ thickness was then spin-coated to form the top cladding layer.

\section{Measurement and Experimental Results}

To characterize the fabricated SiON optical waveguide ring resonator device, the light signal from a broadband amplified spontaneous emission (ASE) light source was made to pass through a polarization controller before it was launched into the input port of the ring resonator via a tapered fiber. The output light signal was then launched into an optical spectrum analyzer (OSA; Agilent 86140B) via another tapered fiber to monitor the spectral response of the ring resonator. Figures $3(\mathrm{a})$ and $3(\mathrm{~b})$ show, respectively, the transmission spectra over the entire $\mathrm{C}$ band for TE and TM mode. It can be seen, for TE and TM mode, that the fabricated ring has the extinction ratios lower than $-18 \mathrm{~dB}$ and the same FSR of $100 \mathrm{GHz}$ but different resonance wavelength. This is because although the effective refractive indices of the ring waveguide are different for TE and TM mode due to birefringence, which results in different resonance wavelength; however, the value of $n_{\text {eff }}$ is usually far less than the circumference $L$ of the ring waveguide. Therefore, the same FSR can be obtained for TE and TM mode for a large radius according to (7). The measured insertion losses are also different for TE and TM mode, and the maximum insertion loss is $9.5 \mathrm{~dB}$ and $8.6 \mathrm{~dB}$ for $\mathrm{TE}$ and TM mode, respectively. The difference is due to the index contrast for TM mode (0.0381) is larger than that for TE mode (0.0365), so the waveguide has more strong constraint for TM mode. In addition, whatever for TE or TM mode, the insertion loss is also slightly different over the entire C band, and the insertion loss at long wavelength $(1570 \mathrm{~nm})$ is somewhat larger than that at short wavelength $(1530 \mathrm{~nm})$. This is from the fact that the mode confinement decreases as the wavelength increases.

The fabricated ring has the full width at half maximum (FWHM) of $0.42 \mathrm{~nm}$ and $0.40 \mathrm{~nm}$, and hence, a finesse of 1.93 for TE mode and 2.03 for TM mode, respectively. Furthermore, the quality factor of the ring resonator is obtained by taking the ratio between the resonance wavelength and the FWHM, and the results are 3700 for TE mode and 3900 for TM mode. From the Q factors, the attenuation in the 


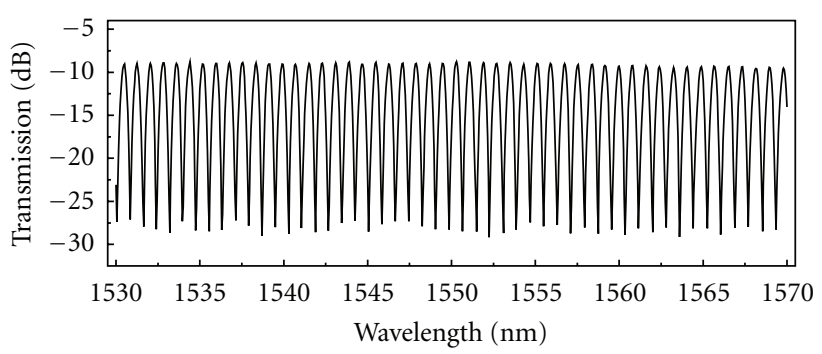

TE

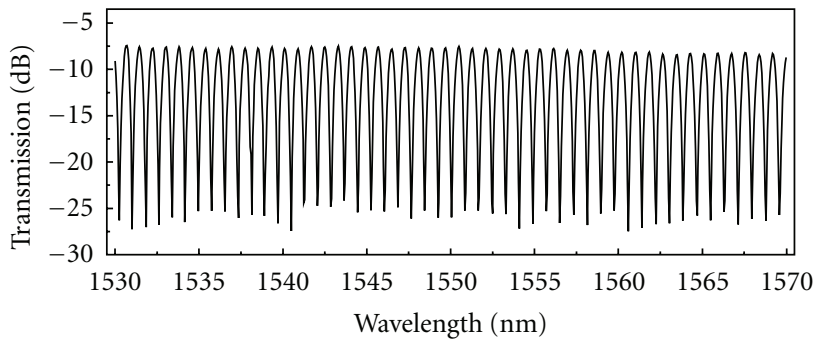

TM

(a)

(b)

FIgURE 3: The measured transmission spectra over the entire C band for TE mode (a) and TM mode (b).

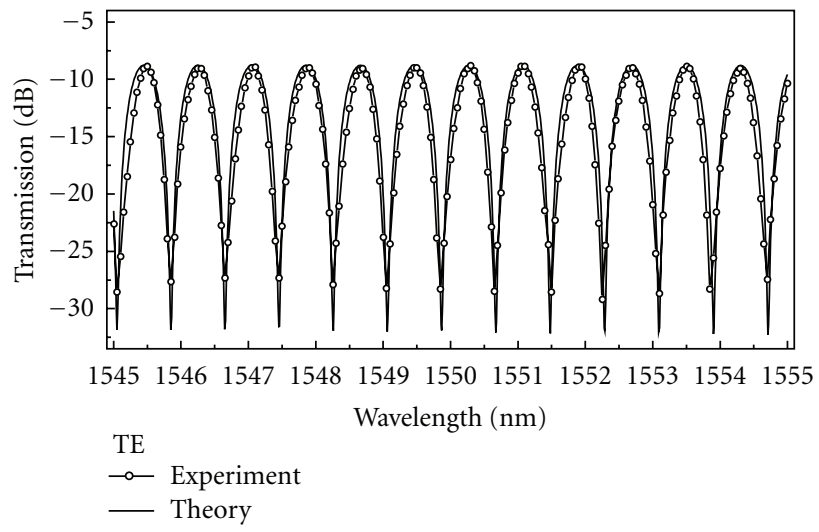

(a)

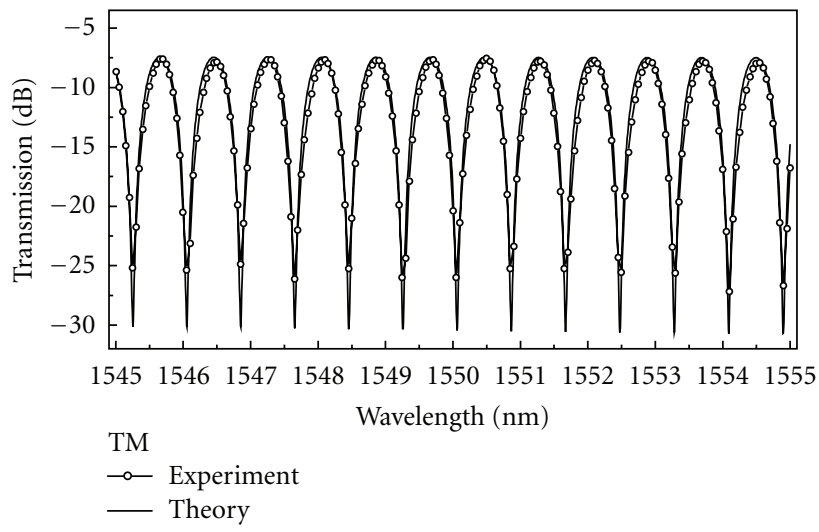

(b)

FIGURE 4: The measured and the simulated transmission spectra over a $10 \mathrm{~nm}$ span for TE mode (a) and TM mode (b).

ring resonator is extrapolated, and we get $18.18 \mathrm{~dB} / \mathrm{cm}$ for TE mode and $17.26 \mathrm{~dB} / \mathrm{cm}$ for TM mode, which correspond to the field attenuation constant $\alpha$ of 0.4543 and 0.4728 , respectively. With the aforementioned parameters, the theoretical response of the ring resonator for TE and TM mode are obtained by using (5) and presented in Figures 4(a) and 4 (b), respectively. It can be seen that there is an excellent agreement between the experimental data and the theoretical one. The fit parameters are $S=24.48 \mathrm{um}$ and $r=301.37 \mathrm{um}$.

\section{Conclusions}

We have designed and fabricated SiON optical waveguide ring resonator by utilizing a TMI to replace the DC in a conventional ring resonator. The critical fabrication tolerance is greatly relaxed and the fabrication process is easy due to using TMI structure. Preliminary results exhibit the same of free spectral range of $100 \mathrm{GHz}$ but different quality factors of 3700 and 3900 at $1550 \mathrm{~nm}$ for TE and TM mode, respectively. The extinction ratio is more than $18 \mathrm{~dB}$ over the entire C-band, and the insertion loss is lower than $9.5 \mathrm{~dB}$ for TE and TM mode. The experimental data of the fabricated ring resonator are also fitted with the theoretical response, and the result show an excellent agreement between them.

\section{Acknowledgments}

This work was supported by the National Natural Science Foundation of China under Grant no. 61177054 and the grant from the Research Grants Council of the Hong Kong Special Administrative Region, China [Project CityU 110507].

\section{References}

[1] O. Schwelb, "Transmission, group delay, and dispersion in single-ring optical resonators and add/drop filters-a tutorial overview," Journal of Lightwave Technology, vol. 22, no. 5, pp. 1380-1394, 2004.

[2] C. K. Madsen and J. H. Zhao, Optical Filter Design and Analysis: A Signal Process Approach, Wiley, New York, NY, USA, 1999.

[3] B. Bhola, H. C. Song, H. Tazawa, and W. H. Steier, "Polymer microresonator strain sensors," IEEE Photonics Technology Letters, vol. 17, no. 4, pp. 867-869, 2005.

[4] C. Y. Chao and L. J. Guo, "Design and optimization of microring resonators in biochemical sensing applications," Journal of Lightwave Technology, vol. 24, no. 3, pp. 1395-1402, 2006.

[5] B. Liu, A. Shakouri, and J. E. Bowers, "Wide tunable double ring resonator coupled lasers," IEEE Photonics Technology Letters, vol. 14, no. 5, pp. 600-602, 2002. 
[6] S. J. Emelett and R. Soref, "Design and simulation of silicon microring optical routing switches," Journal of Lightwave Technology, vol. 23, no. 4, pp. 1800-1807, 2005.

[7] B. E. Little, J. S. Foresi, G. Steinmeyer et al., "Ultra-compact $\mathrm{Si}_{-} \mathrm{SiO}_{2}$ microring resonator optical channel dropping filters," IEEE Photonics Technology Letters, vol. 10, no. 4, pp. 549-551, 1998.

[8] A. Melloni, R. Costa, P. Monguzzi, and M. Martinelli, "Ringresonator filters in silicon oxynitride technology for dense wavelength-division multiplexing systems," Optics Letters, vol. 28, no. 17, pp. 1567-1569, 2003.

[9] P. Rabiei, W. H. Steier, C. Zhang, and L. R. Dalton, "Polymer micro-ring filters and modulators," Journal of Lightwave Technology, vol. 20, no. 11, pp. 1968-1975, 2002.

[10] D. Rafizadeh, J. P. Zhang, S. C. Hagness et al., "Waveguidecoupled AlGaAs/GaAs microcavity ring and disk resonators with high finesse and 21.6-nm free spectral range," Optics Letters, vol. 22, no. 16, pp. 1244-1246, 1997.

[11] K. Honda, E. M. Garmire, and K. E. Wilson, "Characteristics of an integrated optics ring resonator fabricated in glas," Journal of Lightwave Technology, vol. LT-2, no. 5, pp. 714-719, 1984.

[12] R. Germann, H. W. M. Salemink, R. Beyeler et al., "Silicon oxynitride layers for optical waveguide applications," Journal of the Electrochemical Society, vol. 147, no. 6, pp. 2237-2241, 2000.

[13] G. L. Bona, R. Germann, and B. J. Offrein, "SiON highrefractive-index waveguide and planar lightwave circuits," IBM Journal of Research and Development, vol. 47, no. 2-3, pp. 239-249, 2003.

[14] V. D. Nguyen, N. Ismail, F. Sun, K. Wörhoff, T. G. Van Leeuwen, and J. Kalkman, "SiON integrated optics elliptic couplers for Fizeau-based optical coherence tomography," Journal of Lightwave Technology, vol. 28, no. 19, Article ID 5546886, pp. 2836-2842, 2010.

[15] D. J.W. Klunder, F. S. Tan, T. Van der Veen et al., "Experimental and numerical study of SiON microresonators with air and polymer cladding," Journal of Lightwave Technology, vol. 21, no. 4, pp. 1099-1110, 2003.

[16] B. J. Offrein, F. Horst, G. L. Bona, R. Germann, H. W. M. Salemink, and R. Beyeler, "Adaptive gain equalizer in highindex-contrast SiON technology," IEEE Photonics Technology Letters, vol. 12, no. 5, pp. 504-506, 2000.

[17] B. I. Akca, N. Ismail, F. Sun et al., "High-resolution integrated spectrometers in silicon-oxynitride," in Proceedings of the Laser Applications to Photonic Applications, (CLEO '11), OSA Technical Digest, Baltimore, Md, USA, 2011, paper JWA65.

[18] F. B. Veerman, P. J. Schalkwijk, E. C. M. Pennings, M. K. Smit, and B. H. Verbeek, "An optical passive 3-dB TMI-coupler with reduced fabrication tolerance sensitivity," Journal of Lightwave Technology, vol. 10, no. 3, pp. 306-311, 1992.

[19] P. P. Sahu, "A double S-bend geometry with lateral offset for compact two mode interference coupler," Journal of Lightwave Technology, vol. 29, no. 13, pp. 2064-2068, 2011.

[20] F. Rottmann, A. Neyer, W. Mevenkamp, and E. Voges, "Integrated-optic wavelength multiplexers on lithium niobate based on two-mode interference," Journal of Lightwave Technology, vol. 6, no. 6, pp. 946-952, 1988.

[21] T.-Y. Tsai, Z.-C. Lee, J.-R. Chen, C.-C. Chen, Y.-C. Fang, and M. H. Cha, "A novel ultracompact two-mode-interference wavelength division multiplexer for $1.5-\mu \mathrm{m}$ operation," IEEE Journal of Quantum Electronics, vol. 41, no. 5, pp. 741-746, 2005.
[22] B. Li and S. J. Chua, "High carrier injection optical switch based on two-mode interference in SiGe alloy," Applied Physics Letters, vol. 80, no. 2, p. 180, 2002.

[23] B. K. Yang, S. Y. Shin, and D. Zhang, "Ultrashort polarization splitter using two-mode interference in silicon photonic wires," IEEE Photonics Technology Letters, vol. 21, no. 7, pp. 432-434, 2009.

[24] Q. Wu, P. L. Chu, H. P. Chan, and B. P. Pal, "A Y-junction polymer optical waveguide interleaver," Optics Communications, vol. 267, no. 2, pp. 373-378, 2006.

[25] W. Y. Chan, K. X. Chen, H. P. Chan, B. P. Pal, and R. K. Varshney, "A flattop PLC polymer waveguide interleaver based on folded two-stage-cascaded Y-junction Mach-Zehnder interferometers," Optics Communications, vol. 282, no. 5, pp. 883-886, 2009.

[26] K. Chen, H. P. Chan, F. Chen, W. Y. Chan, and B. Sun, "An accurate analysis for two-mode interferometer based MachZehnder interferometers interleaver," Optics Communications, vol. 283, no. 23, pp. 4639-4644, 2010. 


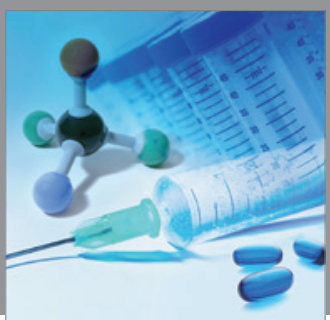

International Journal of

Medicinal Chemistry

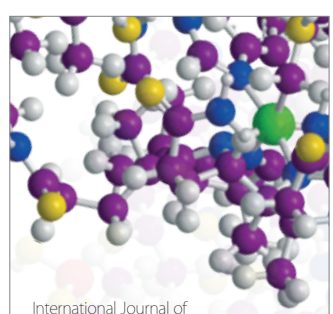

Carbohydrate Chemistry

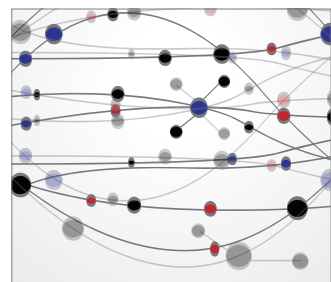

The Scientific World Journal
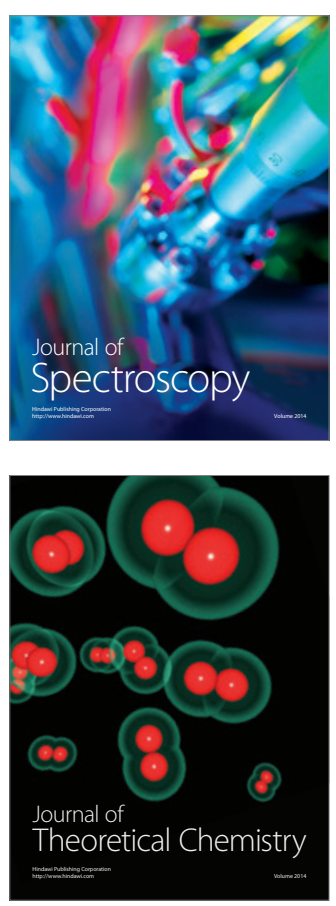
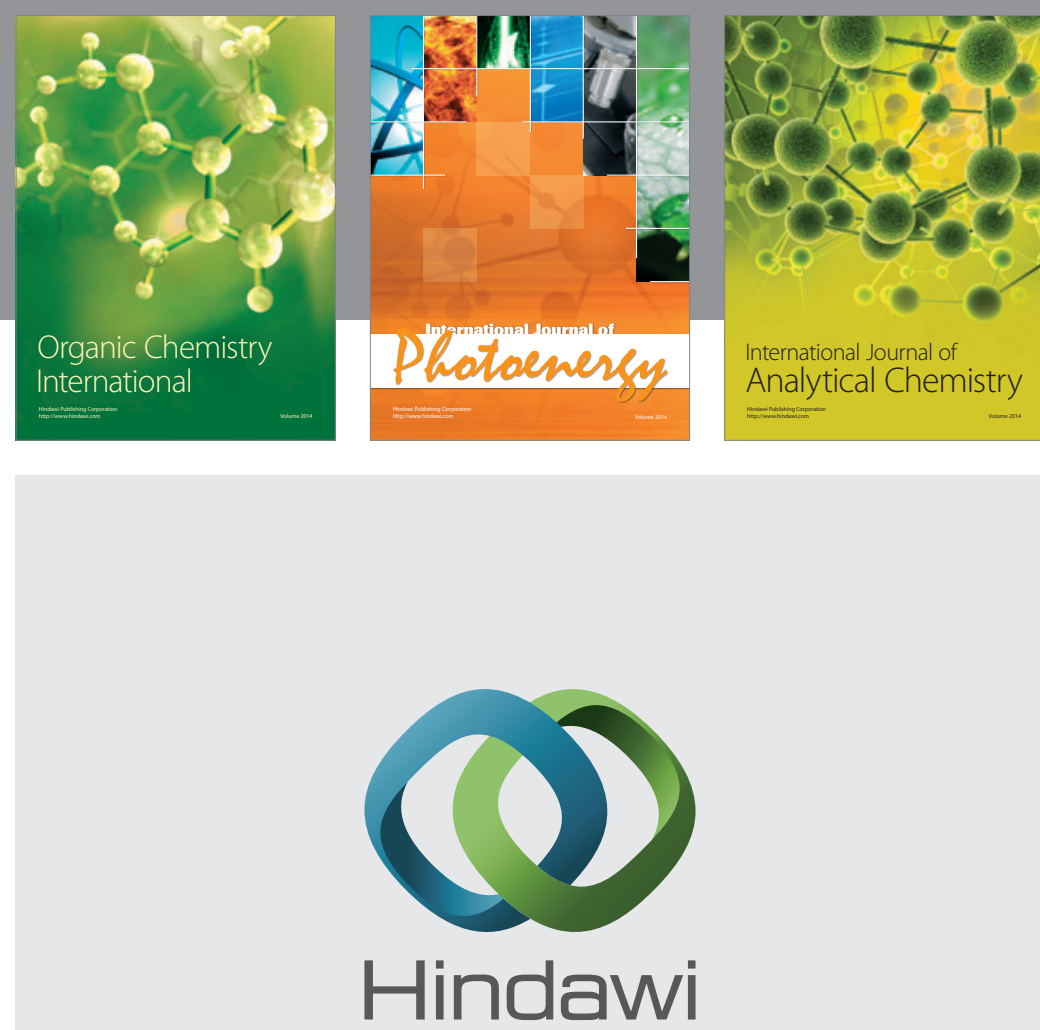

Submit your manuscripts at

http://www.hindawi.com
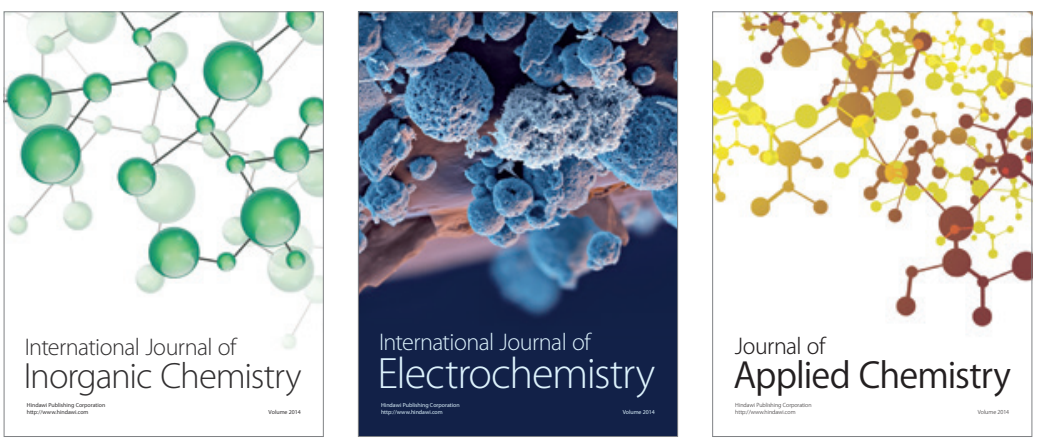

Journal of

Applied Chemistry
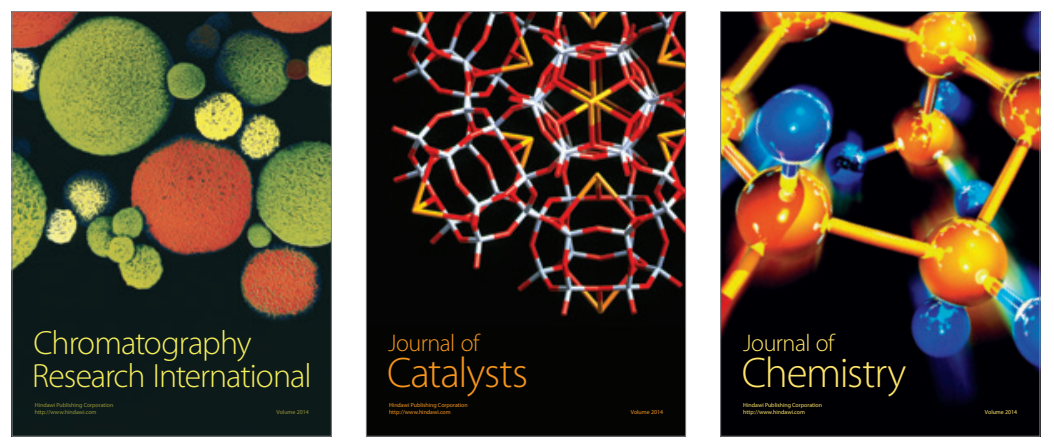
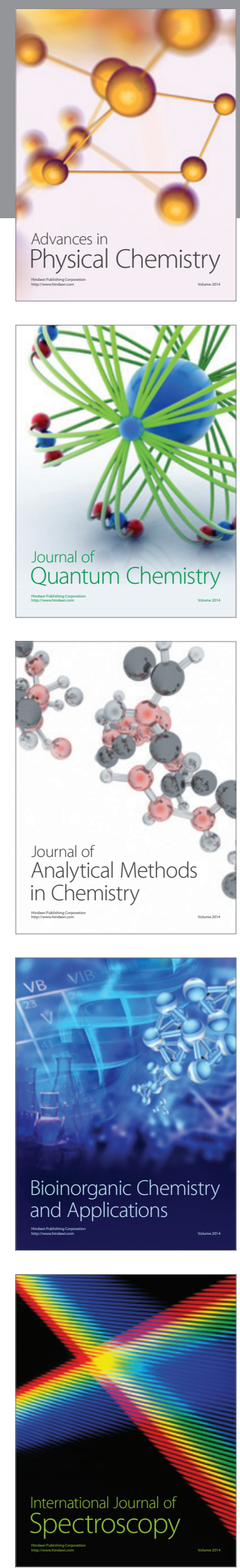